\title{
Simultaneous optimization of inclination angle and fin characteristics on heat transfer in a rectangular enclosure
}

\author{
Samira Payan****, Sameh A. Nada** and A. H. Sarhaddi* \\ * Department of mechanical engineering, Shahid Nikbakht Faculty of Engineering, University of Sistan and Baluchestan, Zahedan, Iran \\ ** Department of Mechanical Engineering, Benha Faculty of Engineering, Benha University, Benha 13512 \\ ***Corresponding Author : s_payan_usb@eng.usb.ac.ir
}

$\begin{array}{ll}\text { Submitted } & : 19 / 07 / 2019 \\ \text { Revised } & : 24 / 12 / 2019 \\ \text { Accepted } & : 19 / 02 / 2020\end{array}$

\begin{abstract}
This paper examines the prospect of increasing or decreasing the heat transfer through a rectangular enclosure with an aspect ratio of 2 in two Rayleigh numbers $10^{5}$ and $10^{6}$ by optimizing its inclination angle and the parameters of a highly conductive thin fin attached to its hot surface. The enclosure is heated from below, and the dominant heat transfer mechanism within the enclosure is the free convection. Optimization is performed by particle swarm optimization algorithm. The equations of energy, continuity, and momentum for free convection heat transfer in the enclosure are discretized by the finite volume method and are numerically solved. The optimization objective is to adjust the enclosure inclination angle and the fin parameters (position and height), so that heat transfer into the cold wall becomes minimized or maximized. The attained results reveal that the heat transfer through such an enclosure can be increased significantly up to $23 \%$ by its inclination angle $20.6^{\circ}$ and the addition of a thin fin at the optimal location 0.72 and optimal height 0.2 in Rayleigh $10^{6}$. Also, the obtained results show that heat transfer can be decreased up to $32 \%$ by the slight adjustment of its inclination angle $1.7^{\circ}$ and addition of a thin fin at the optimal location 1.41 and optimal height 0.2 in Rayleigh number $10^{5}$.
\end{abstract}

Keywords: Free convection, PSO algorithm, Optimal height, Optimal position, Optimal angle.

\section{INTRODUCTION}

Free convection is a well-known and well-studied mechanism of the heat transfer and plays a critical role in many engineering systems and applications such as electronic devices, thermal insulations, thermal design (Alshayji et. Al, 2019), ventilation of closed spaces, crystal growth, chemical reactions, and fabrication of semiconductors. Thus, when dealing with such systems and applications, free convection heat transfer needs to be examined with great care. Free convection heat transfer in enclosures has been the subject of many types of research, which have examined the issue from different angles and with different approaches. Also, one of the main objects in many papers is the investigation of the enhancement or reduction of free convection heat transfer in the enclosures with the attached fins. Accordingly, previous literature associated with this object has been reviewed in the following section. 
The finite difference technique in order to probe the natural convection heat transfer in a rectangular cavity was utilized (Hasnaoui et al., 1992) that is locally heated from below. In their study, the top surface is kept cold at a constant temperature $T_{c}$, and the bottom surface is partially heated. They also examined the periodic and nonperiodic solutions for Rayleigh numbers' ranges higher than the critical value. The effect of the boundary conditions on free convection heat transfer in a rectangular cavity was studied (Corcione, 2003) that was heated from below and cooled from above. The impacts of different aspect ratios and Rayleigh numbers on the flow field and heat transfer were studied. The effect of different aspect ratios and inclination angles on free convection heat transfer in inclined cavities also was explored (Rahman and Sharif, 2003). The natural convection heat transfer in inclined rectangular cavities with infinity conductive fins attached to the hot wall was considered (Lakhal et al., 1997). In this work, the studies were carried out with Rayleigh numbers of $10^{4}$ to $2 \times 10^{5}$, aspect ratios of 2.5 to infinity, dimensionless fin length of 0 to 1 , dimensionless fin spacing of 0.33 to aspect ratio, enclosures inclination angles of 0 to 60 degrees, and Prandtl number of 0.72 . They stated that heat transfer is minimized when dimensionless fin length is 0.75 and dimensionless fin spacing is 0.33 and decreases with the increase of enclosure inclination angle. The influence of adding a thick fin ( $0.5 \%$ to $10 \%$ of enclosure width) with a length of $20 \%$ of enclosure width on heat transfer performance was explored (Nag et al., 1994). The effect of attached fins to the heated surface of a passive solar massive system was experimentally investigated (Bilgen, 2001). Free convection, radiation, and conduction heat transfer in the wall were considered. The results showed that about $40 \%$ of the radiation heat flux on the fins surface went through the system and dissipated at the black. A free convection flow and heat transfer mechanism between two concentric circular tubes was experimentally studied (Nada, 2008). A range of Rayleigh numbers and inclination of the annulus and different spaces between two circular tubes were investigated in their works. The results showed that the rise of gap widths and Rayleigh numbers enhances heat transfer, and an increase in the inclination angle from the horizon decreases heat transfer.

The effect of an arrangement of heat fins attached to the heat wall of vertical and horizontal slender cavities on free convection flow field and heat transfer in a range of Rayleigh number less than $3 \times 10^{5}$ was investigated (Nada, 2007). He expressed that, in the design of electronic components, there are solid motivations to mount as many electronic components as possible in a given enclosure. This leads to high power generation density, and this can increase the temperature of the packages above the allowable limit. To conquer this challenge, the rate of heat transfer from packages should be maximized. The results showed that optimum fin spacing existed, at which heat transfer maximized. The effect of ventilation opening slots in the sides and top walls of cavities heated from below on the cooling of the cavities was experimentally studied (Nada and Moawad, 2004). The experimental studies were carried out for a range of inclination angles of the cavity 0 to 180 and different slots opening ratios of $1,0.75$, and 0.25 . The experimental studies showed that Nusselt numbers decrease with the increase of tilt angle in any slots' ratios also in each inclination angle with the increase of slots' ratios. Nusselt numbers increase for the top-venting arrangement. Moreover, their results showed that, for side-venting and side and top venting with the increase of tilt angle to 90 , Nusselt numbers increased in each opening ratio and then decreased from tilt angle 90 to 180. A correlation of average Nusselt number for free convection heat transfer in opening slots' enclosures from their experimental studies is obtained (Nada and Elattar, 2016). The correlation is relative to many effective parameters including Rayleigh number, opening ratios, and inclination angles. The importance of the free convection heat transfer mechanism in enclosure motivated several researchers to optimize the effect of the different parameters on the problem. The PSO algorithm to determine the optimal parameters of an array of thin fins and a fin grooved and inclined fin attached to the hot wall of a confined cavity wherein free convection is the dominant heat transfer mechanism, respectively, was used (Azimifar and Payan, 2017; Dindarloo and Payan, 2019). They assumed that the horizontal surfaces of the cavity be inactive and its vertical side walls be active. In their work, the range of Rayleigh numbers was between $10^{4}$ and $10^{6}$ in their work. The prospect of increasing the heat transfer through the confined cavities by applying geometric change based on the concept of highly conductive solid blocks was studied (Azimifar and Payan, 2016). In this study, the confined cavity was examined with three sets of boundary conditions. They showed that the very conductive blocks attached to the walls could change the shape of the geometry of the confined cavity. The range of Rayleigh 
number was between $10^{4}$ and $10^{5}$ in their work. The influence of optimal length of two fins attached to the insulated walls of a confined cavity with active vertical walls and insulated horizon the all walls (Zhang et al., 2016) was exhibited and used the conjugate gradient algorithm to reduce the heat transfer of the cavity with the fins of a constant length. As can be seen, optimization of position and dimensions of thin fins within an enclosure is a well-researched subject. It can show the importance of enclosures heated from below and the effect of fin parameters on the heat transfer mechanism within these enclosures. The present paper is focused on this type of enclosure and the effect of its inclination angle on the rate of heat transfer, into the cold wall. To the best of our knowledge, simultaneous optimization of height and position of a thin fin on the horizontal hot wall of enclosures and tilt angle of enclosures with free convection has not been carried out until now. Also, in all of the previous literatures, heat transfer decrease in high Rayleigh numbers was not detected, while high conductive thin fins attached to the hot wall were detected. But, this paper shows that very conductive fins can decrease heat transfer when those are set in optimal position with an optimal height. In this paper, the PSO algorithm (PSOA) (Eberhart and Kennedy, 1995) has been selected for obtaining optimal fin parameters and the inclination angle of cavity, because this algorithm is very simple and powerful for the finding of few optimization parameters as we regard in this paper (3 parameters:1-fin height, 2-fin position, and 3-the inclination angle of the cavity). This algorithm also used in other applications for its simplicity (Al-Gharabally et al.,2021). The goal of optimization is to decrease or increase the free convection heat transfer from cold wall in a cavity with aspect ratio equal to 2 . Some application reasons are caused to select aspect ratio equal 2; for example, optimization for a solar collector is very time consuming, because the aspect ratio is very high; therefore, some researchers (Amraqui, 2011) considered aspect ratios 0.5, 1, and 2 for it. In the current paper, we considered the largest aspect ratio among them, that is, 2. Furthermore, in this aspect ratio, the length of active walls is greater than inactive walls; therefore, for many applications such as cooling of electronic devices, it is suitable. Besides, reconstruction of high aspect ratio cavities for electronic device cooling is not simple, and the Nu number in them is lower than the average $\mathrm{Nu}$ number in the small aspect ratios. In order to numerically solve the set of momentum, energy equations, and continuity in this cavity, the finite volume technique is employed, and in this way, SIMPLER approach for solving the mentioned equations is utilized. The fins are attached on the horizontal hot wall in the cavity. This paper is divided into two important parts. In the first part of this study, a 2D numerical model for free convection heat transfer in a rectangular enclosure heated from below and cooled from above with a thin fin attached to its hot surface was developed. The investigation is performed with Rayleigh numbers $\mathrm{Ra}=10^{5}$ and $\mathrm{Ra}=10^{6}$ and enclosure inclination angles in the range of $\theta=0-20^{\circ}$ (this narrow range of inclination angle is considered to free convection from below hot wall to be dominant). Fin position and height are obtained by an optimization algorithm, and the role of enclosure inclination angle on the fin position is studied. In this part, in each fixed tilt angle, PSO algorithm chooses a couple of coordinates $\left(\mathrm{X}_{\text {fin }}, \mathrm{Y}_{\text {fin }}\right)$ for every fin, such that $\mathrm{Y}$ determines height of fin, and $\mathrm{X}$ represents the fin position. The fin is attached to the hot wall of the cavity and coincides with grids of the domain. It is important to note that nondimensional diffusion coefficients in energy and momentum equations are taken as infinity in order to model a high conductive thin fin on the hot wall. In the second part of the study, the PSO algorithm is used to optimize the thin fin parameters and enclosure inclination angle simultaneously with the objective of increasing/decreasing the heat transfer rate. In this part, a tilt angle for the enclosure and a pair of coordinates $\left(\mathrm{X}_{\text {fin }}, \mathrm{Y}_{\text {fin }}\right)$ for the fin are selected by the PSO algorithm, and the optimization values for the parameters are obtained. The results show that very conductive thin fins can change the shape of streamlines and decrease heat transfer from a heated cavity from below.

\section{DESCERIPTION OF THE PROBLEM}

The physical model of a 2D rectangular enclosure filled with an incompressible fluid is illustrated in Figure 1. The dominant heat transfer mechanism within the cavity is free convection governed by Boussinesq approximation. The cavity has a length of $\mathrm{L}$, the height of $\mathrm{H}$, and an aspect ratio of $2(\mathrm{AR}=2)$; its bottom surface is heated to temperature $t_{h}$, its top surface is cooled to temperature $t_{c}$, and both of its sidewalls are insulated. Radiation heat transfer in the cavity is neglected, because we assume that the surfaces temperature values are not very high. The aim of this problem is to maximize or minimize $\overline{\mathrm{Nu}} \mathrm{e}, \mathrm{av}$. The objective function is defined as 
$\mathrm{G}\left(\mathrm{x}_{\text {fin }}, \mathrm{y}_{\text {fin }}, \mathrm{q}\right)=\mathrm{Nu}_{\mathrm{e}, \mathrm{av}}\left(\mathrm{x}_{\text {fin }}, \mathrm{y}_{\text {fin }}, \mathrm{q}\right)$

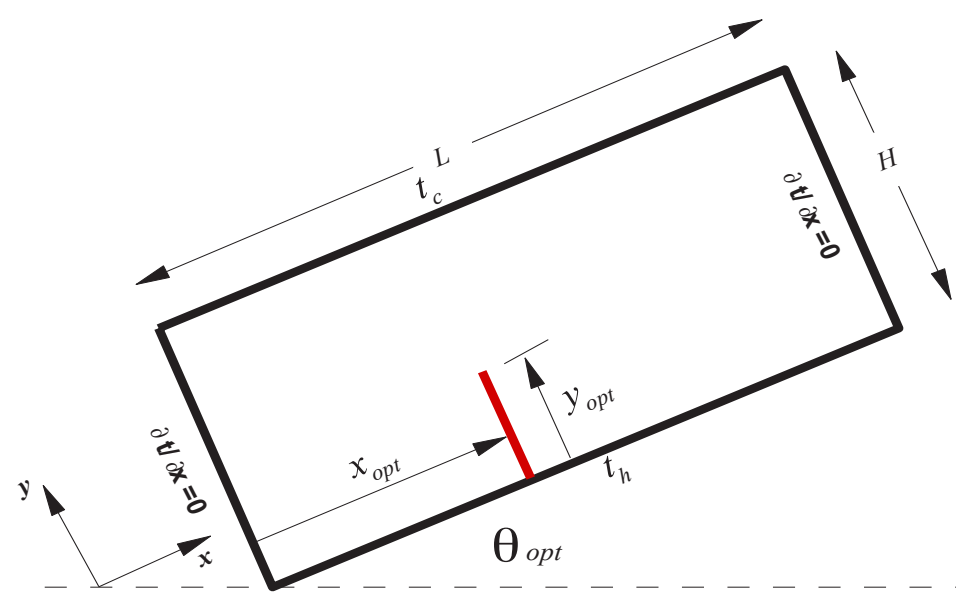

Figure 1. The schematic figure of a rectangular cavity with optimal parameters of thin fins attached on the hot wall and optimal inclination angle.

In Eqs. (2), the height of the straight fin $\left(\mathrm{x}_{\text {fin }}\right)$ and its position $\left(\mathrm{y}_{\text {fin }}\right)$ and the angle of inclination $q$ (counted counterclockwise from the horizontal) are unknown. Those are design variables. The constraints of each design variable are applied as follows:

\begin{tabular}{|c|c|}
\hline $0.0 \leq \theta \leq \pi / 9$ & (2a) \\
\hline $0.01 \mathrm{~L} \leq \mathrm{x}_{\text {fin }} \leq 0.99 \mathrm{~L}$ & (2b) \\
\hline $0.01 \mathrm{H} \leq \mathrm{y}_{\text {fin }} \leq 0.2 \mathrm{H}$ & (2c) \\
\hline
\end{tabular}

The usual PSO algorithm (Payan et.al, 2015) is used in this paper. To solve this problem, the unknown coordinates of

$\left(\mathrm{x}_{\mathrm{fin}}, \mathrm{y}_{\mathrm{fin}}\right)$ for the thin fin are considered on the hot wall. In order to solve the governing equations via the control volume method, the fin is fixed in the closest position consistent with grids. The thick of fin is Dx . In the governing equations, nondimensional diffusion parameters set infinite values to consider very conductive thin fin.

Because fin is very conductive, then an attached very conductive fin changes the shape of the hot boundary surface (Azimifar and Payan, 2016). To speed up the resolution and accuracy of the PSO algorithms, and since a fin with high conductivity is considered to minimize the heat transfer, the maximum height fin is considered $0.2 \mathrm{H}$. Also, for dominance of natural heat transfer from the cavity heated from below, the angle of inclination is selected in the range of $0-\pi / 9$. 


\section{MATHEMATICAL MODELING AND GOVERNING EQUATIONS}

The problem of free convection heat transfer can be solved with a series of nonlinear partial differential equations of continuity, energy, and momentum conservation. For this particular type of free convection problem, energy equations are joined with momentum equations via Boussinesq method:

$$
\rho=\rho_{0}\left[1-\beta\left(t-t_{0}\right)\right]
$$

The nondimensional forms of the governing equations are given as follows:

$$
\begin{gathered}
\nabla . \mathbf{V}=0.0 \\
\mathbf{V} . \nabla^{*} \mathbf{V}=\nabla^{*} \mathrm{P}+\Gamma \nabla^{* 2} \mathbf{V}+\mathrm{Grg} \\
\mathbf{V} . \nabla^{*} \mathrm{~T}=\Gamma^{\prime} \nabla^{* 2} \mathrm{~T}
\end{gathered}
$$

where $\mathrm{G}$ is 1.0 for the fluid domain and infinity for the solid domain. $\mathrm{G} \phi \mathrm{is}^{-1}$ is the fluid domain and infinite for very conductive solid. A very conductive solid attached to the surface causes a new shape of surface to exist. Here, the nondimensional variables are as follows:

$$
\begin{gathered}
\nabla^{*}=(\nabla / \mathrm{L}) ; \mathbf{V}=(\mathbf{v L} / \mathrm{v}) ; \mathrm{T}=\mathrm{t}_{-} \mathrm{t}_{\mathbf{c}} /(\Delta \mathrm{t}) \\
\mathbf{P}=\left(\mathbf{p}+\rho_{0}(\mathbf{g . r})-\mathrm{p}_{0}\right) / \rho_{0}(v / \mathrm{L})^{2}
\end{gathered}
$$

And dimensionless groups of Grashof, Prandtl, and Rayleigh are defined as follows:

$$
\mathrm{Gr}=\mathrm{gbDtH} / u^{2} ; \operatorname{Pr}=u / a ; \mathrm{Ra}=\operatorname{PrGr}
$$

The boundary conditions of the given partial differential equations are

$$
\begin{gathered}
\mathrm{U}=0.0, \mathrm{~V}=0.0, \mathrm{~T}(\mathrm{X})=0.5 \text { at } \mathrm{Y}=0 \\
\mathrm{U}=0.0, \mathrm{~V}=0.0, \mathrm{~T}(\mathrm{X})=-0.5 \text { at } \mathrm{Y}=1 \\
\mathrm{U}=0.0, \mathrm{~V}=0.0,(\nabla \mathrm{T}) \mathbf{e}_{1}=0.0 \text { at } \mathrm{X}=0,2
\end{gathered}
$$


Equations 4a-c are solved numerically through the finite volume technique. Also, a staggered grid is used to discretize the velocity equation and take the effect of the velocity field into consideration. The power-law scheme is employed to discretize displacement and diffusion terms, and the iterative SIMPLER algorithm is used to solve velocity-pressure dependent equations. Detailed explanation about this method can be found in Patankar (1980). The Nusselt numbers of the cold wall are calculated as follows:

$$
\begin{gathered}
\overline{\mathrm{Nu}}_{\mathrm{e}, \mathrm{av}}=1 / 2 \sum_{\mathrm{nd}=1}^{\mathrm{R}} \mathrm{Nu}_{\mathrm{e}, \mathrm{nd}} \Delta \mathrm{X} \\
\text { where } \\
\left.\overline{\mathrm{Nu}}_{\mathrm{e}, \mathrm{nd}}=(\nabla \mathrm{Te})_{2}\right)_{\mathrm{nd}}, \mathrm{nd}=1,2, . ., \mathrm{R}
\end{gathered}
$$

where $\overline{\mathrm{Nu}}_{e, a \mathrm{v}}$ denotes the estimated Nusselt number, for the cold wall.

\section{GRID INDEPENDENCE AND VALIDATION MODEL}

We considered a rectangular enclosure heated from below and cooled from above. Vertical surfaces are assumed to be insulated $(\theta=0.0)$. The cavity is filled with an incompressible fluid with Prandtl number $\operatorname{Pr}=0.71$. The goal is to assess the effect of mesh on the average Nusselt number of the hot wall for Rayleigh numbers $\mathrm{Ra}=105$ and $\mathrm{Ra}=10^{6}$.

The average Nusselt numbers are obtained for the hot wall via different meshes and Rayleigh numbers, which are compared in Figure 2. The results show that a uniform $120 \times 90$ mesh has the best performance regarding the accuracy and computational time. In Table 1, these results are compared with the results of Corcione (2003). As can be seen, the error is less than $3 \%$.

Table 1. Comparison of average Nusselt numbers.

\begin{tabular}{|c|c|c|c|}
\hline Ra & Present work & Corcione, 2003 & Relative Error \% \\
\hline $10^{6}$ & 7.45 & 7.35 & 1.36 \\
\hline $10^{5}$ & 4.41 & 4.31 & 2.32 \\
\hline
\end{tabular}




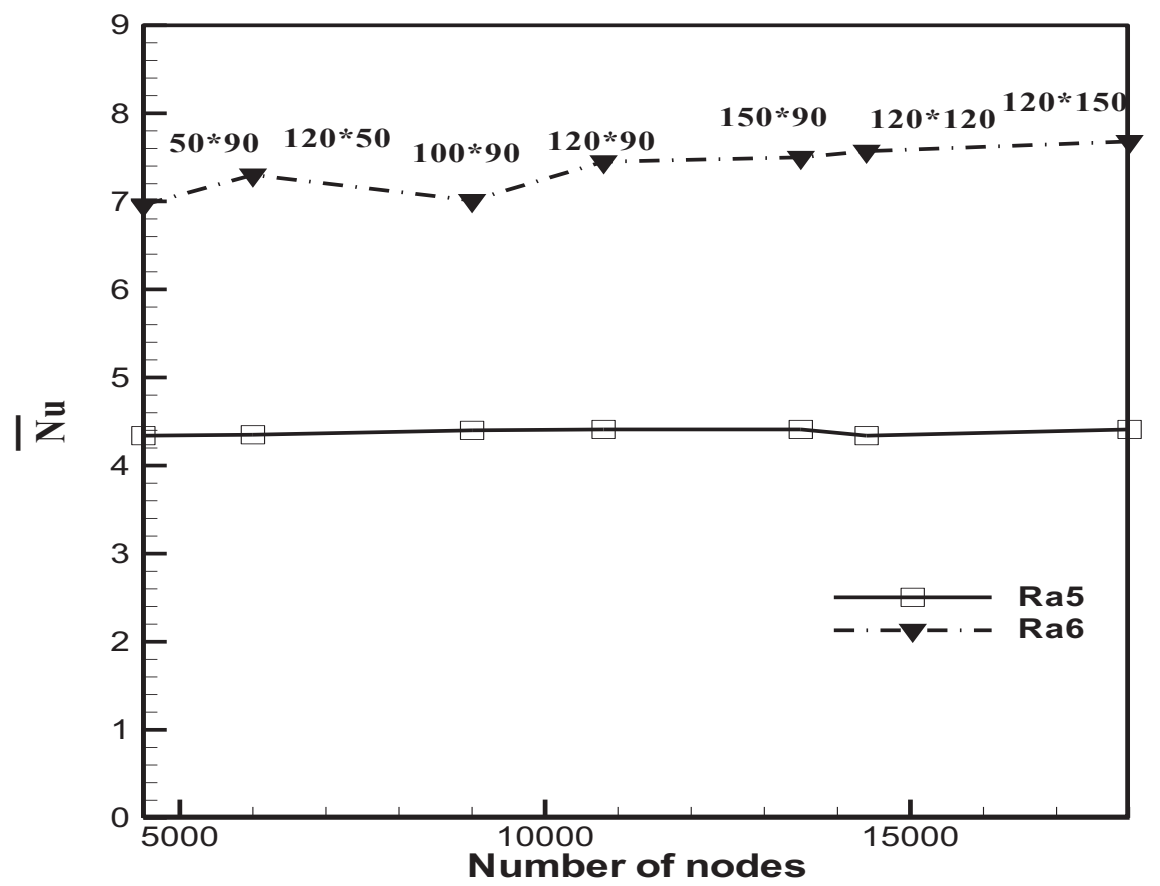

Figure 2. Comparison of the average Nusselt number on the hot wall of the rectangular cavity, which is heated from the bottom.

To validate the model in the case of inclination of the enclosure, the average Nusselt numbers under different angles are compared with the results of Rahman and Sharif (2003), which were deduced for similar conditions (Rayleigh numbers $\mathrm{Ra}=2 \times 10^{5}$, Prandtl number $\operatorname{Pr}=0.71$, and aspect ratio $A R=2$ ) and experimentally validated in different ranges of conditions (Nada and Moawad, 2004; Nada and Elattar, 2016). The error of the present analysis at different inclination angles is shown in Table 2. As seen, the maximum errors are less than $4 \%$ for all cases. Besides, the maximum errors for the inclination angles between 0 and 30 are less than $2 \%$, that is, a good agreement with Rahman and Sharif (2003).

Table 2. The comparison of its average Nusselt numbers obtained from Rahman and Sharif (2003) and the present work.

\begin{tabular}{|c|c|c|c|}
\hline angle & $\begin{array}{c}\text { Rahman and Sharif, } \\
\mathbf{2 0 0 3}\end{array}$ & Present work & Relative Error \% \\
\hline 15 & 4.87 & 4.81 & 1.23 \\
\hline 30 & 5.05 & 5 & 1 \\
\hline 45 & 5.36 & 5.21 & 2.8 \\
\hline 60 & 5.57 & 5.36 & 3.77 \\
\hline
\end{tabular}




\begin{tabular}{|c|c|c|c|}
\hline 75 & 5.56 & 5.37 & 3.41 \\
\hline 90 & 5.45 & 5.24 & 3.85 \\
\hline
\end{tabular}

\section{RESULTS AND DISCUSSION}

In the following sections, the results of individual and simultaneous optimization of the fin height /position and the inclination angle of the enclosure are presented.

\subsection{Optimization of the Fin Height/Position And Angle of İnclination}

The considered rectangular enclosure in this study is assumed to have $\mathrm{AR}=2$ and cooled from above and heated from below. It can be modeled as a part of a solar collector or a cavity with electronic device. The enclosure is inclined toward the horizon and has two insulated sidewalls and a highly conductive thin fin, which is attached to its hot surface as shown in Figure 1. The goal is to obtain the optimal fin height and position on the hot wall at different enclosure inclination angles, so that heat transfer into the cold wall becomes minimized or maximized. The optimization results obtained for different inclination angles at Rayleigh numbers $\mathrm{Ra}=10^{5}$ and $\mathrm{Ra}=10^{6}$ are shown in Tables 3 and 4, respectively.

The percentage of increase and decrease achieved with these Rayleigh numbers demonstrates the algorithm's ability for estimation an optimal fin position and height for the considered inclination angles. The flow contours obtained with $\mathrm{Ra}=10^{5}$ when the objective is to increase the heat transfer are shown in Figure 3. Also, temperature distributions for this study can be seen in supplementary data (Figure S 1).

Table 3. Thin fin parameters and reducing and increasing the heat transfer for Rayleigh $10^{5}$ at different angles of rectangular cavity.

\begin{tabular}{|c|c|c|c|c|}
\hline effect & $\mathbf{X}_{\text {Opt }}$ & $\mathbf{Y}_{\text {Opt }}$ & $\boldsymbol{\theta}$ & $\mathbf{N u}_{\text {no-fin }} / \mathbf{N u}_{\text {fin }}$ \\
\hline decrease & 1.41 & 0.2 & 0 & $4.4 / 2.99=1.47$ \\
\hline increase & 1.01 & 0.2 & & $4.4 / 4.69=0.947$ \\
\hline decrease & 0.82 & 0.16 & 5 & $3.9 / 3.25=1.20$ \\
\hline increase & 1 & 0.2 & & $3.9 / 4.68=0.833$ \\
\hline decrease & 0.78 & 0.15 & & $3.98 / 3.41=1.167$ \\
\hline increase & 0.78 & 0.2 & 10 & $3.98 / 4.74=0.840$ \\
\hline
\end{tabular}




\begin{tabular}{|c|c|c|c|c|}
\hline decrease & 0.74 & 0.2 & \multirow{2}{*}{15} & $4.07 / 3.47=1.173$ \\
\hline increase & 1.0 & 0.2 & & $4.07 / 4.57=0.891$ \\
\hline decrease & 0.83 & 0.2 & \multirow{2}{*}{20} & $4.14 / 3.62=1.143$ \\
\hline increase & 1.8 & 0.2 & & $4.14 / 4.25=0.974$ \\
\hline
\end{tabular}

As can be seen in the inclination angles of 0 and $5^{\circ}$, locating the fin at the center of the surface creates two large vortices, but when the inclination angle increases to 10 degrees, the number of vortices rises to 3 , and the heat transfer rate increases.

Table 4. Parameters of the thin fin and the decrease and increase of the heat transfer for Rayleigh $10^{6}$ at different angles of the rectangular cavity.

\begin{tabular}{|c|c|c|c|c|}
\hline effect & $\mathbf{X}_{\mathbf{O p t}}$ & $Y_{\text {Opt }}$ & $\theta$ & $\mathbf{N u}_{\text {no-fin }} / \mathbf{N u}_{\text {fin }}$ \\
\hline decrease & 0.64 & 0.1 & \multirow{2}{*}{0} & $7.45 / 5.08=1.46$ \\
\hline increase & 0.94 & 0.2 & & $7.45 / 8.12=0.917$ \\
\hline decrease & 0.64 & 0.11 & \multirow{2}{*}{5} & $6.08 / 5.18=1.174$ \\
\hline increase & 0.70 & 0.2 & & $6.08 / 8.78=0.692$ \\
\hline decrease & 0.57 & 0.18 & \multirow{2}{*}{10} & $6.27 / 5.27=1.190$ \\
\hline increase & 0.72 & 0.2 & & $6.27 / 9.01=0.696$ \\
\hline decrease & 0.60 & 0.2 & 15 & $6.99 / 5.75=1.216$ \\
\hline
\end{tabular}




\begin{tabular}{|c|c|c|c|c|}
\hline increase & 0.74 & 0.2 & & $6.99 / 9.17=0.762$ \\
\hline decrease & 0.49 & 0.16 & & $7.08 / 6.04=1.172$ \\
\hline increase & 0.69 & 0.2 & & $7.08 / 9.19=0.770$ \\
\hline
\end{tabular}

At inclination angles less than $10^{\circ}$, a fin, which is attached to positions other than the center (at almost $x=0.8$ ), can also create three vortices, but they will not be as strong as the two vortices created when the fin is positioned at the center.
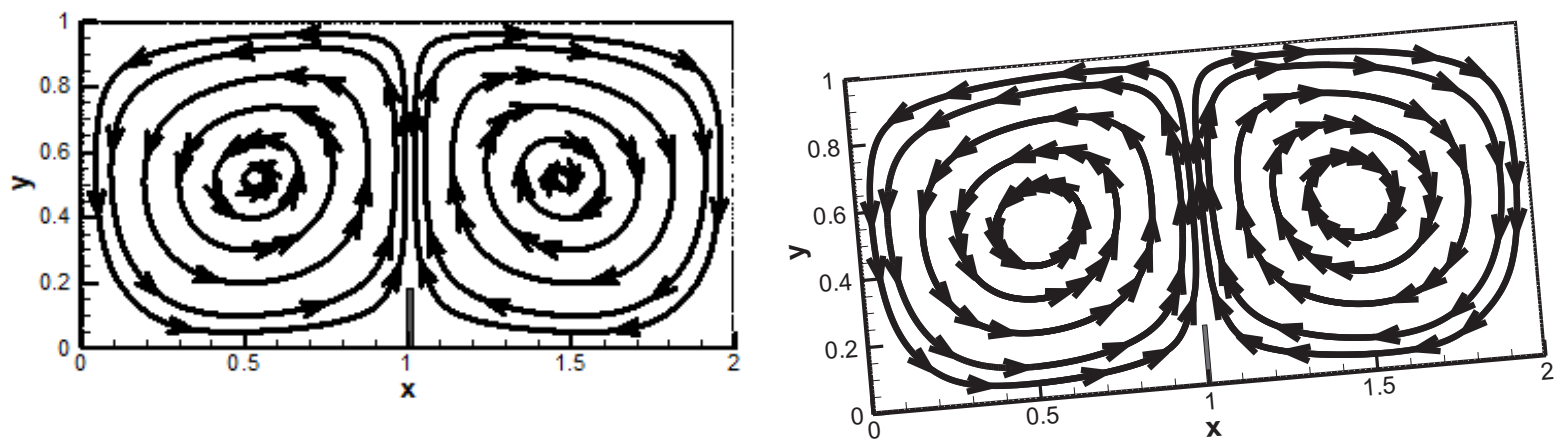

$$
\theta=0^{\circ}
$$

$$
\theta=5^{\circ}
$$
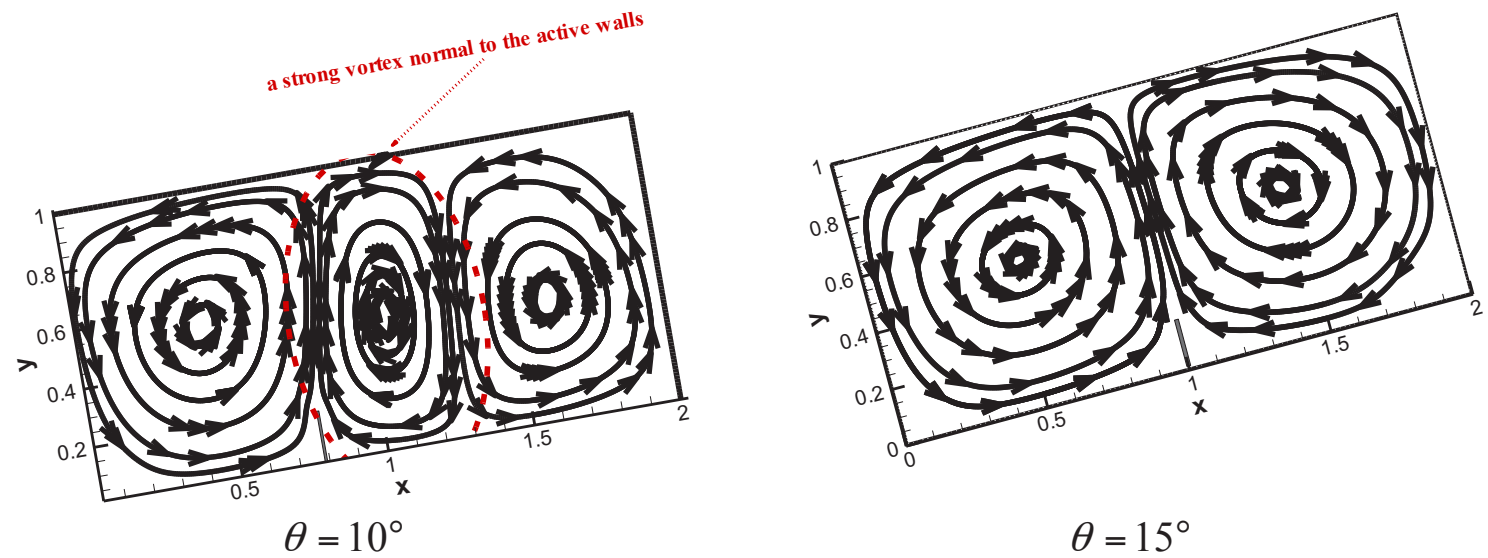


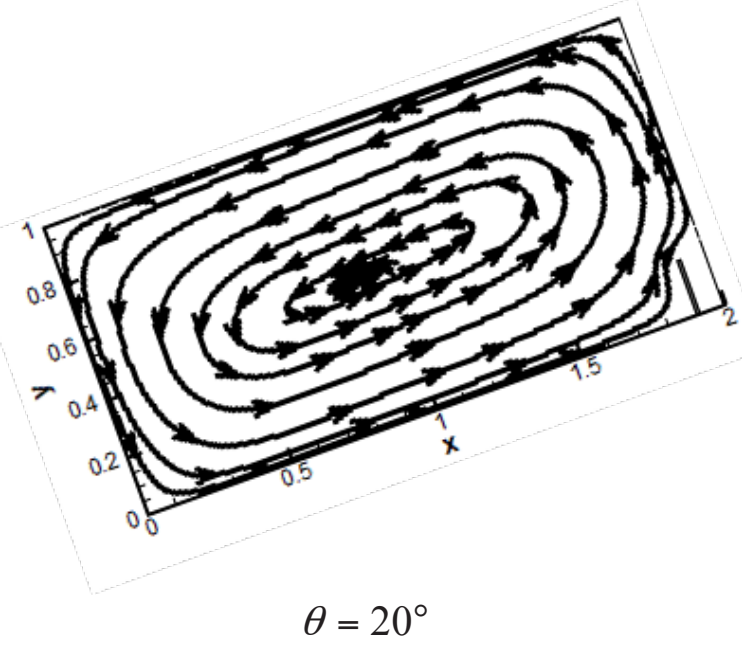

Figure 3. Streamlines for the increase of heat transfer using PSOA in five incline angles $0,5,10,15$ and $20^{\circ} \mathrm{f}$ or $\mathrm{Ra}=10^{5}$.

At inclination angles between 10 and $15^{\circ}$, again, two large vortices exist and account for the greatest value of heat transfer. At inclination angle of $20^{\circ}$, a single large vortex is responsible for the greatest share of heat transfer rate.

As seen, for the cooling of a hot device in each of the inclination angles, the best fin height is $0.2 \mathrm{H}$. Table 3 shows that, in every inclination angle, the fin height chosen by the PSO algorithm to increase the heat transfer is the maximum value allowed under the considered conditions. This is not an unexpected result, because to maximize the heat transfer, the fin with the highest possible conductivity and the largest possible height must be attached to the hot surface. Therefore, for the cooling of a hot device in each of the inclination angles, the best fin height is $0.2 \mathrm{H}$. When $\mathrm{Ra}=10^{5}$ and the objective is to decrease the heat transfer, in every inclination angle, the optimal fin position is $\mathrm{x}=0.8$, which leads to the creation of small attenuating vortices near the hot wall and a large vortex covering the entire enclosure. Contrary to the previous state, when the objective was to increase the heat transfer with the existence of two or three strong vortices, here, only a single weak vortex emerges. At inclination angle of $0^{\circ}$, the optimal fin position is far from the center, and it leads to the greatest reduction in the rate of heat transfer. As seen in Figure 4, the optimal fin position has led to dominant longitudinal large vortex that increases boundary layer thickness on the cold wall. Also, temperature distributions for this study can be seen in supplementary data (Figure S 2). A notable result of Table 3 is that despite the objective (decreasing the heat transfer), the optimal fin height is still $0.2 \mathrm{H}$ in three cases, which indicates that, with this fin height, fin's role in decreasing the heat transfer by obstructing the flow still surpasses the contribution of its high conductivity to heat transfer. Although the highest absolute Nusselt number is observed at the inclination angle of $10^{\circ}$, as Table 3 shows, the effect of fin peaks when enclosure inclination angle is $5^{\circ}$. When the objective is to decrease the heat transfer, both the highest heat transfer reduction and the greatest fin impact occur at the angle of $0^{\circ}$. Furthermore, the results show that a step shape of hot surface can decrease heat transfer. 


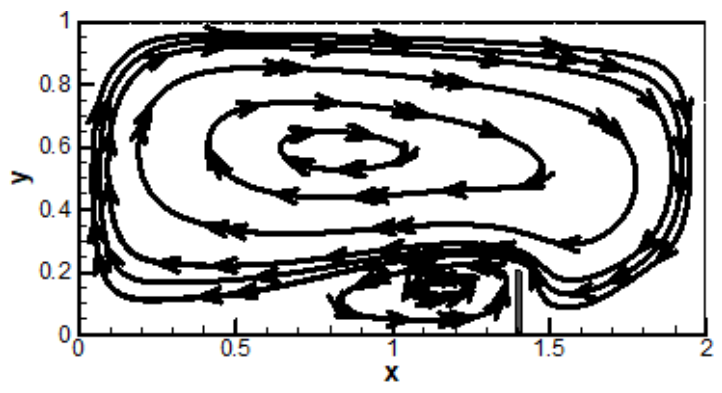

$\theta=0^{\circ}$

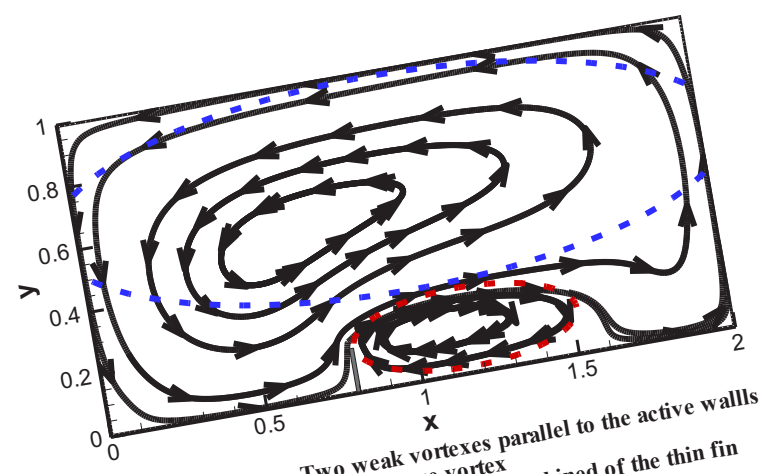

Two weak vortexes parale

--

$\theta=10^{\circ}$

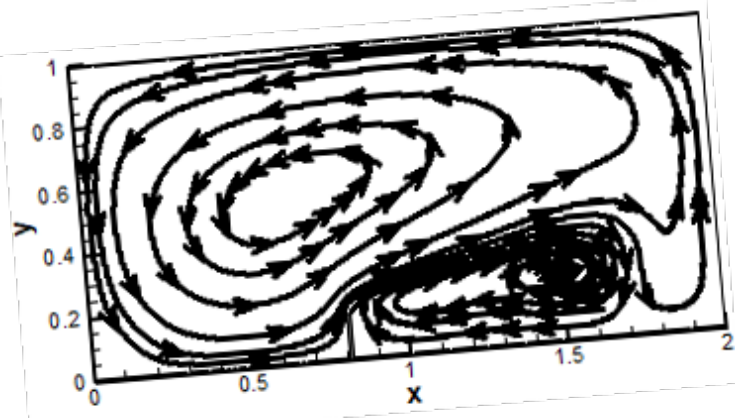

$$
\theta=5^{\circ}
$$

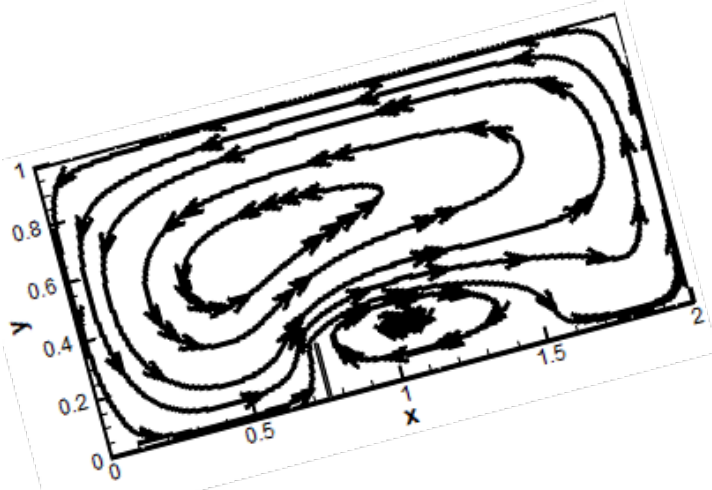

$\theta=15^{\circ}$

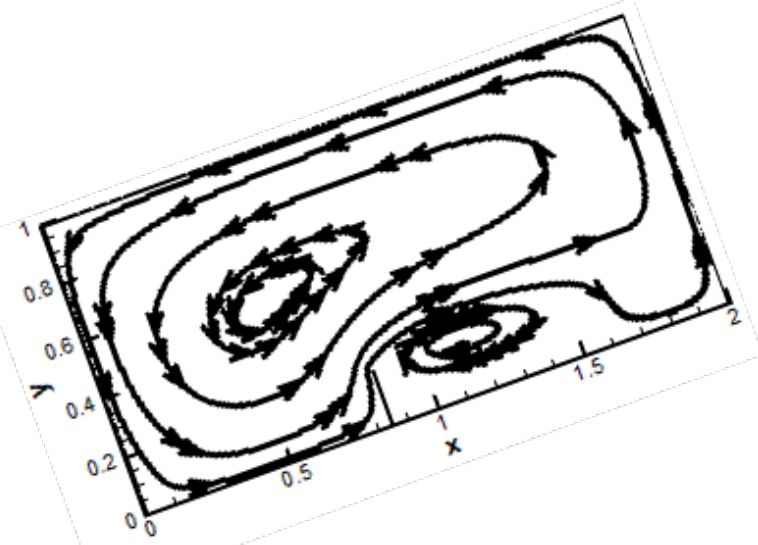

$$
\theta=20^{\circ}
$$


This type of geometry can be used in solar collectors. Figure 5 shows the flow contours obtained with $\mathrm{Ra}=10^{6}$ when the objective is to increase the heat transfer. Also, temperature distributions for this study can be seen in supplementary data (Figure S 3). As can be seen, in every inclination angle between 5 and $20^{\circ}$, attachment of the fin (at $\mathrm{x}=0.7$ ) creates three large vertical vortices, but at inclination angle of $0^{\circ}$, two large vortices exist and account for the greatest share of heat transfer. As Table 4 shows, at every inclination angle, the fin height chosen by the PSO algorithm to increase the heat transfer is again the maximum value allowed under the considered conditions. This table also shows that, for a fixed fin position, the heat transfer rate increases with the increase of enclosure inclination angle. The difference between Figure 3 and Figure 5 indicates that when the objective is to increase the heat transfer rate, emergence of additional and larger vertical vortices with $\mathrm{Ra}=10^{6}$ ensures and enhances heat transfer, but with $\mathrm{Ra}=10^{5}$ this enhancement is not guaranteed. When $\mathrm{Ra}=10^{6}$ and the objective is to decrease the heat transfer, at every inclination angle, the optimal fin position falls between $\mathrm{x}=0.5$ and $\mathrm{x}=0.65$, which leads to the emergence of small attenuating vortices near the hot wall and a large vortex that is parallel to hot and cold surfaces and spans over the entire enclosure. Again, contrary to the previous state when the goal was to increase the heat transfer and two or three strong vortices were created, here, only a dominant single weak vortex emerges. This phenomena can be seen in Figure 6. Also, temperature distributions for this study can be seen in supplementary data (Figure S 4). As Figure 6 shows, the optimal fin position has led to dominant longitudinal large vortex that increases boundary layer thickness on the cold wall. It is notable that, unlike the results obtained with $\mathrm{Ra}=10^{5}$, where the optimal fin height was found to be the maximum allowed value $(0.2 \mathrm{H})$, the optimal fin height obtained with $\mathrm{Ra}=10^{6}$ is $0.1 \mathrm{H}$. This is due to the reduction in the thickness of the boundary layer, and it increased high velocity flows that come close to the hot wall. The results of Table 4 also show that when the objective is to increase the heat transfer, the highest absolute Nusselt number occurs at enclosure inclination angle of $20^{\circ}$, but in $\mathrm{Ra}=10^{5}$, highest absolute Nusselt number occurs at enclosure inclination angle of $5^{\circ}$.

\subsection{Simultaneous Optimization of the Inclination Angle And Fin Parameters}

In this case, the considered rectangular enclosure with $\mathrm{AR}=2$ is also cooled from above and heated from below. This enclosure has two insulated sidewalls, and a highly conductive thin fin is attached to its hot surface. The cavity inclination angle is unknown. The optimal fin height and position and optimal enclosure inclination angle to increase/decrease heat transfer when $\mathrm{Ra}=10^{5}$ and $\mathrm{Ra}=10^{6}$ are unknown are shown in Figure 1 . In this problem, the cavity inclination angle and the fin parameters are optimized simultaneously. As Table 5 shows, for both Rayleigh numbers, the best inclination angle to decrease the heat transfer is about zero $\left(1.7^{\circ}\right)$. However, when the objective is to increase the heat transfer, the best inclination angles for $\mathrm{Ra}=10^{5}$ and $\mathrm{Ra}=10^{6}$ are 10.87 and $20.6^{\circ}$, respectively. For comparison of optimization and nonoptimization run time problems, a case is expressed in this part, for maximization of heat transfer using optimal angle of cavity and fin parameters by a laptop (intel (R) core (TM) i7-6700 HQ CPU (a) 2.6GH, Ram $8 \mathrm{~GB}$ ), for each iteration, run time is 1 hour and 30 minutes, but for a cavity without optimization, run time is $1 \mathrm{~min}$ and 30 seconds. 
Table 5. Parameters of thin fin and the decrease and the increase of the heat transfer for Rayleigh number $10^{5}$ and $10^{6}$ at the angle of rectangular cavity optimization.

\begin{tabular}{|c|c|c|c|c|c|}
\hline $\mathbf{R a}$ & $\mathbf{X}_{\text {Opt }}$ & $\mathbf{Y}_{\text {Opt }}$ & $\boldsymbol{\theta}_{\text {Opt }}$ & \%decrease/\%increase & $\overline{\mathbf{N u}}_{\text {no-fin }} / \overline{\mathbf{N u}}_{\text {fin }}$ \\
\hline $10^{5}$ & 1.41 & 0.2 & 1.71 & $32.95 /----$ & $4.4 / 2.95=1.49$ \\
\hline $10^{5}$ & 0.77 & 0.2 & 10.87 & $----/ 7.95$ & $4.4 / 4.75=0.926$ \\
\hline $10^{6}$ & 0.6 & 0.1 & 1.70 & $32.39 /-----$ & $7.47 / 5.05=1.48$ \\
\hline $10^{6}$ & 0.72 & 0.2 & 20.60 & $-----/ 23.29$ & $7.47 / 9.21=0.81$ \\
\hline
\end{tabular}

The maximum horizontal and vertical nondimensional velocities and the maximum magnitude of nondimensional velocity vectors and their positions are reported in Table 6 . As can be seen, when the aim of heat transfer is the enhancement, the maximum horizontal velocities locate near the cold wall, and the maximum magnitude of velocity vectors was greater than when the aim of heat transfer is depression. The maximum horizontal velocities locate near the fin attached to the hot wall for the goal of decrease of heat transfer. Therefore, it is shown that fin blocking is accrued.

Table 6. Parameters of velocity (maximum values of vertical and horizontal velocities and the maximum magnitude of velocities) in the best conditions for two Rayleigh numbers reported in Table 5.

\begin{tabular}{|c|c|c|c|c|c|c|}
\hline$\tilde{x}$ & 窇 & $\mathbf{X}$ & $\mathbf{Y}$ & $\mathbf{U}_{\max }$ & $\mathbf{V}_{\max }$ & $|\mathbf{V}|_{\text {max }}$ \\
\hline \multirow{6}{*}{$\because$} & \multirow{3}{*}{ 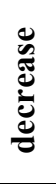 } & 1.347 & 0.301 & -147.6 & - & - \\
\hline & & 1.449 & 0.210 & - & 104.5 & - \\
\hline & & 1.415 & 0.267 & - & - & 154.0 \\
\hline & \multirow{3}{*}{$\begin{array}{l}\stackrel{\Xi}{\Xi} \\
\stackrel{\Xi}{\circlearrowright} \\
. \Xi\end{array}$} & 0.500 & 0.903 & -159.6 & - & - \\
\hline & & 1.297 & 0.460 & - & -197.2 & - \\
\hline & & 1.297 & 0.460 & - & - & 197.6 \\
\hline \multirow{6}{*}{$\stackrel{\circ}{\circ}$} & \multirow{3}{*}{ 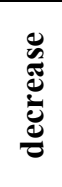 } & 0.6695 & 0.176 & 551.9 & - & - \\
\hline & & 1.941 & 0.608 & - & 393.5 & - \\
\hline & & 0.6525 & 0.176 & - & - & 562.5 \\
\hline & \multirow{3}{*}{ 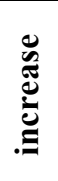 } & 0.4661 & 0.949 & -488.5 & - & - \\
\hline & & 1.076 & 0.415 & - & -592.8 & - \\
\hline & & 1.076 & 0.415 & - & - & 594.5 \\
\hline
\end{tabular}




\section{CONCLUSION}

In this paper, the effects of the inclination angle and the position and height of the fin attached to the hot bottom surface of a rectangular enclosure were investigated and optimized to decrease or increase the heat transfer rate. Individual and simultaneous optimizations of said angle and fin parameters on the heat transfer through the enclosure are considered. The results showed that as the Rayleigh number changes from $\mathrm{Ra}=10^{5}$ to $\mathrm{Ra}=10^{6}$, the optimal fin position shifts towards lower X's. It was also found that the fin is more effective in increasing the heat transfer when $\mathrm{Ra}=10^{6}$ than when $\mathrm{Ra}=10^{5}$. But when the objective is to decrease the heat transfer, the fin has the same effect in both Rayleigh numbers. The results also indicate that while reducing the heat transfer in the enclosure heated from two surfaces requires the use of lowly conductive fins, for a cavity heated from below, a small fin can alter the shape of vortices and thereby significantly decrease the heat transfer, provided that its height and position are optimized by a fitting optimization procedure.

\section{REFERENCES}

Alshayji, A. \& Ebrahim, S. 2019. Numerical Simulation of Heat Transfer Process in Inclined Roofs with Radiant Barrier System. Journal of Engineering Research. 8(2): 305-323.

Hasnaoui, M., Bilgen, E. \& Vasseur, P. 1992. Natural convection heat transfer in rectangular cavities partially heated from below. J. Thermophys. Heat Trans. 6(2): 255-264.

Corcione, M. 2003. Effect of thermal boundary conditions at sidewalls upon natural convection in rectangular enclosures heated from below and cooled from above, Int. J. Therm. Sci., 42(2): 199-208.

Rahman, M. \& Sharif, M. A. R. 2003. Numerical study of laminar natural convection in inclined rectangular enclosures of various aspect ratios, Num. Heat Trans., 44(2): 355-373.

Lakhal, E.K., Hasnaoui, M., Bilgen, E. \& Vasseur, P. 1997. Natural convection in inclined rectangular enclosures with perfectly conduction fins attached on the heated wall, Heat Mass Trans., 32(5): 365-373.

Nag, A., Sarkar, A. \& Sastri, V.M.K. 1994. Effect of thick horizontal partial partition attached to one of the active walls of a differentially heated square cavity, Num. Heat Trans., 25(5): 611-625.

Bilgen, E. 2001. Experimental study of massive wall systems with fins attached on the heated wall and with glazing, Heat Mass Trans. 38(1): 159-164.

Nada, S.A. 2008. Experimental Investigation of natural convection heat transfer in horizontal and inclined annular fluid layer, Heat Mass Trans., 44: 929-936.

Nada, S.A. 2007. Natural convection heat transfer in horizontal and vertical closed narrow enclosure with heated rectangular finned base plate, Int. J. Heat Mass Trans., 50(3): 667-679.

Nada, S.A. and Moawad, M. 2004. Free convection in tilted rectangular enclosures heated at the bottom wall and vented by different slot-venting arrangements, Exp. Therm. Fluid Sci. 28(8): 853-862.

Nada, S.A. and Elattar, H.F. 2016, Experimental investigation and general correlation of passive heat transfer in enclosures at different operating, orientations and venting configurations, Appl. Therm. Eng., 102(5): 346-358, 2016.

Azimifar, A. \& Payan, S. 2017. Optimization of characteristics of an array of thin fins using PSO algorithm in confined cavities heated from a side with free convection, Appl. Therm. Eng., 110: 1371-1388.

Dindarloo, M. R. and Payan, S. 2019. Effect of fin thickness, grooves depth, and fin attachment angle to the hot wall on maximum heat transfer reduction in a square enclosure, Int. J. Therm. Sci. 136: 473-490.

Azimifar A. \& Payan, S. 2016. Enhancement of heat transfer of confined enclosures with free convection using blocks with PSO algorithm, Appl. Therm. Eng. 101(25): 79-91. 
Zhang, D.D., Zhang, J.H., Liu, D., Zhao F.Y. \& Wang, H.Q. 2016. Conjugate thermal transport enhancement for an air filled enclosure with heat conducting partitions using inverse convection methodology, Int. J. Heat Mass Trans., 102: 788-800.

Eberhart, R.C. \& Kennedy, J. 1995. Particle swarm optimization, paper presented at, Proc. of the Sixth Int. Symposium on Micro Machine and Human Science.

Al-Gharabally, M., F. Almutairi, A. \& A. Salman, A. 2021. Particle swarm optimization application for multiple attribute decision making in vertical handover in heterogenous wireless networks. Journal of Engineering Research. 9(1): 176-187.

Amraqui, S., Mezrhab, A. \& Abid, C. 2011. Combined natural convection and surface radiation in solar collector equipped with partitions, Appl. Sol. Energy, 47: 36-47.

Payan, S., Sarvari, S.M.H \& Farahmand, A. 2015. Inverse boundary design radiation problem with radiative equilibrium in combustion enclosures with PSO algorithm, Int. Commun. Heat Mass Trans. 68: 150-157.

Patankar, S. V. 1980. Numerical Heat Transfer and Fluid Flow, hemisphere, Washington DC, USA. 